\title{
Is nuclear viscosity dependent on temperature?
}

\author{
E. Vardaci ${ }^{1, \star}$, A. Di Nitto ${ }^{2}$, P.N. Nadtochy ${ }^{3}$, G. La Rana1 ${ }^{1}$, M. Cinausero ${ }^{4}$, G. Prete ${ }^{4}$, N. Gelli ${ }^{5}$, E.M. \\ Kozulin $^{6}$, G.N. Knyazheva ${ }^{6}$, I.M. Itkis ${ }^{6}$, M. Ashaduzzaman ${ }^{1}$, F. Davide ${ }^{1}$, A. Pulcini ${ }^{1}$, D. Quero ${ }^{1}$, \\ and P.K. Rath ${ }^{1,7}$ \\ ${ }^{1}$ Istituto Nazionale di Fisica Nucleare and Diparimento di Fisica "E. Pancini", Universitá degli Studi di Napoli \\ "Federico II", Napoli, 80126, Italy \\ ${ }^{2}$ Johannes Gutenberg- Universität Mainz Institut für Kernchemie, Mainz, Germany \\ ${ }^{3}$ Omsk State Technical University, Mira prospekt 11, 44050 Omsk, Russia \\ ${ }^{4}$ Laboratori Nazionali di Legnaro dell'Istituto Nazionale di Fisica Nucleare, Legnaro (Padova), 35020, Italy \\ ${ }^{5}$ Istituto Nazionale di Fisica Nucleare, Sesto Fiorentino (Firenze), 50019, Italy \\ ${ }^{6}$ Flerov Laboratory of Nuclear Reactions, Joint Institute for Nuclear Research, RU-141980 Dubna, Moscow \\ Region, Russia \\ ${ }^{7}$ Manipal Centre For Natural Sciences, Manipal University, 576104, India
}

\begin{abstract}
.
Nuclear viscosity is an indispensable ingredient of the nuclear fission collective dynamical models. It governs the exchange of energy between the collective variables and the thermal bath. Its dependence on the shape and temperature is a matter of controversy. By using systems of intermediate fissility we have demonstrated in a recent study that the viscosity parameters is larger for compact shapes, and decreases for larger deformations of the fissioning system, at variance with the conclusions of the statistical model modified to include empirically viscosity and time scales. In this contribution we propose an experimental scenario to highlight the possible dependence of the viscosity from the temperature.
\end{abstract}

\section{Introduction}

It is well known that the large amplitude collective motion occurring in nuclear fission is an ideal laboratory for probing nuclear dissipation $[1,2]$. Experimental evidences on how nuclear viscosity affects the compound fission process, as well as the competition with the fusion-evaporation process, has come from the observation of the evolution of many observables such as prescission multiplicities of neutrons, charged particles and $\gamma$-rays with the excitation energy. Broadly speaking, these quantities appear to be significantly larger than those predicted by the statistical model. The main important consequence of fission viscosity is to slow down the collective motion toward the separation in two fragments with the implication that prescission radiations are more likely to escape. The bulk results are an increase of their multiplicity and a higher probability of producing evaporation residues. Applications of the statistical model which include, with a specific ansatz, the effects of viscosity give rather controversial estimates of the time scale and strength of viscosity [3-9].

\footnotetext{
^e-mail: vardaci@na.infn.it
} 

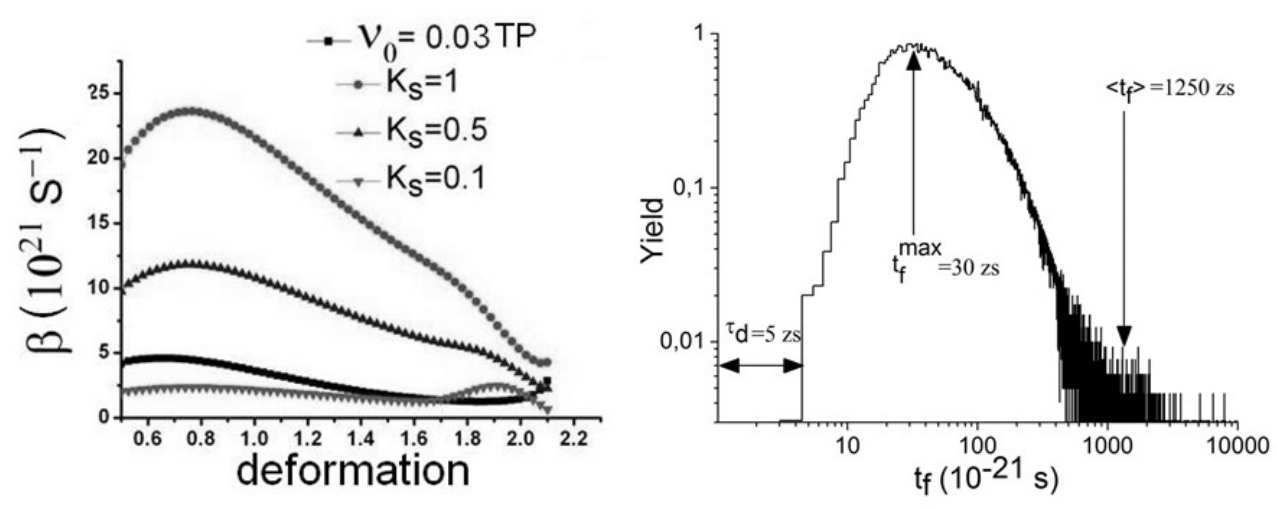

Figure 1. Main results of the 3D calculations for the system ${ }^{32} \mathrm{~S}+{ }^{100} \mathrm{Mo}$ at $200 \mathrm{MeV}$. Left:Reduced viscosity parameter vs. deformation of the compound nucleus. $\mathrm{K}_{s}$ is the strength of the one-body dissipation. Full strength is for $\mathrm{K}_{s}=1$. The black solid line is the functional dependency expected in the case of the two-body dissipation. Right:Calculated distribution of the fission times.

Indeed, a dynamical picture of the fission process affected by viscosity cannot be effectively included in the statistical model. This matter has been discussed in some detail in [9]. Dynamical models can include dissipation effects due to viscosity and fluctuations ab-initio [10-14]. In the past years we have developed a 3D dynamical model based on the Langevin approach to fission to calculate the decay of light particles from the fissioning nucleus $[14,15]$. We have applied this model to our extensive set of data gathered on systems of intermediate fissility $[8,15]$ and have extracted a more convincing picture of the nature of dissipation and its dependence on the evolving shape of the nucleus.

The two main results are summarized in figure 1. The viscosity parameter is found to be strongly dependent on the shape of the nucleus undergoing fission, being larger for compact shapes (around the equilibrium configuration) and smaller at the more elongated shapes (shapes around the saddle point and more deformed). In particular, our set of data agrees with the one-body nature of dissipation represented by $\mathrm{K}_{s}=1$ (full strength). One unprecedented outcome of our model is the calculation of the time distribution of the fission events (figure 1, right). This distribution typically extends over 3 orders of magnitude and takes into account multiple chance fission. The time delay parameter widely used in the statistical approach does not correspond to any of the characteristic times of this distribution. This result confirms the inadequacy of the statistical model approach to nuclear dissipation. The wide extension of the time distribution found also explains why different time scales are extracted within statistical model implementations of nuclear viscosity when different probes are used. The results of this work [15] have further implications on the dynamical evolution of composite systems in other non-compound processes like quasifission [16].

\section{How to probe a temperature-dependent viscosity}

In the above calculations nuclear viscosity is assumed independent from the temperature. The nuclear mechanism of dissipation may indeed depend on the temperature. A pathway to explore such a dependence was undertaken by the following observations. In many experimental studies on heavy systems 

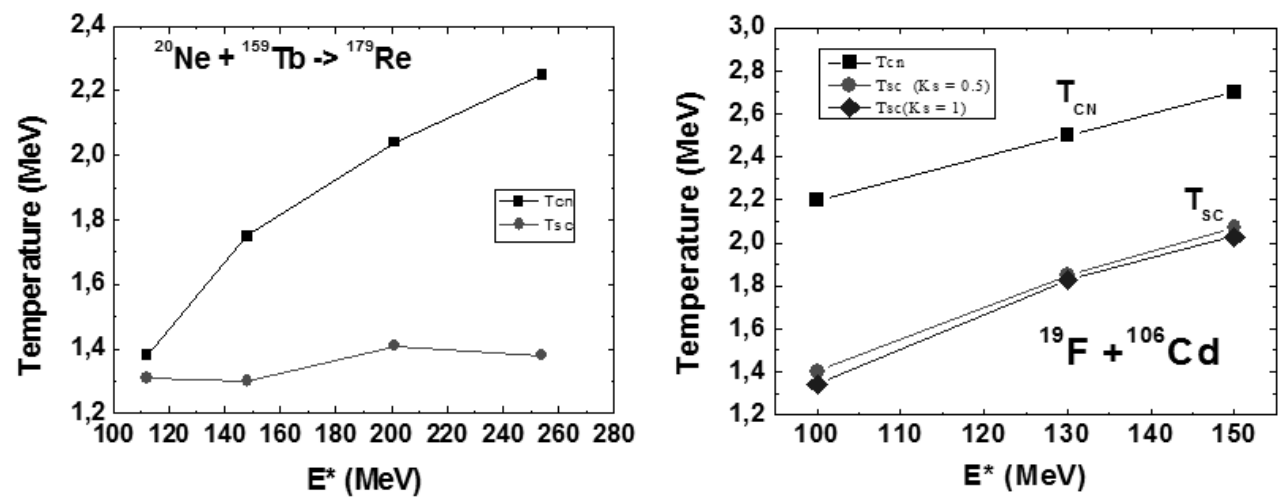

Figure 2. Left:Pre- and post-scission temperatures vs. initial excitation energy of the compound nucleus [17]. Right:Pre- and post-scission temperatures vs. initial excitation energy of the compound nucleus in the case of an intermediate mass system calculated with a dynamical model [14]. $\mathbf{K}_{s}=1$ is for full one-body dissipation.

it is commonly found that if the temperature of the compound nucleus $\mathrm{T}_{C N}$ is raised, the temperature at scission point $\mathrm{T}_{S C}$ remains almost constant $[1,17,18]$. This supports the picture in which fission occurs after that the composite nucleus has cooled down until it has reached an excitation energy of roughly $50-60 \mathrm{MeV}$, independently on the excitation energy of the initial compound nucleus. This is shown in figure 2 (left) [17].

If a study on the dependence of nuclear viscosity on the temperature is performed with this picture in mind (for instance by measuring the excitation function of some observables of the kind above), the measurable effects on the observables, which are deduced from the deviations from the model used, are averaged over an interval of temperatures $\Delta \mathrm{T}=\mathrm{T}_{C N}-\mathrm{T}_{S C}$ which grows with the bombarding energy. In other words, as the interval of temperatures $\mathrm{T}_{C N}-\mathrm{T}_{S C}$ increases, the observable effects are integrated over a larger and larger interval of temperatures. A possible dependence on the temperature will be therefore hidden. Consequently, heavy systems, like the one in figure 2 (left), are not the ideal frame for the study of the dependence of the viscosity parameter from the temperature.

Systems of intermediate fissility have instead a completely different behavior. With our model [14] we calculated $\mathrm{T}_{S C}$ for several systems. In figure 2 (right) we show the dependence of $\mathrm{T}_{C N}$ and $\mathrm{T}_{S C}$ from the initial excitation energy of the compound nucleus for the case of the system ${ }^{19} \mathrm{~F}+{ }^{106} \mathrm{Cd}$. The interesting feature of these systems is that $\mathrm{T}_{S C}$ also grows with the excitation energy of the initial compound nucleus, a behavior at variance with that found for heavier systems. This is due to the delicate balance between the time scale of the fission process and the binding energy of the pre-scission particles: the neutron pre-scission emission, which is mainly responsible for the cooling in the pre-saddle region, is suppressed by the higher binding energy and stronger competition with charged particles. As a result, the two fission fragments retain most of the initial excitation energy.

\section{Experiment and results}

An experiment was designed to probe the dependence of the nuclear viscosity on temperature within the picture above, keeping in mind that in case we are unable to reproduce a large set of data with our 


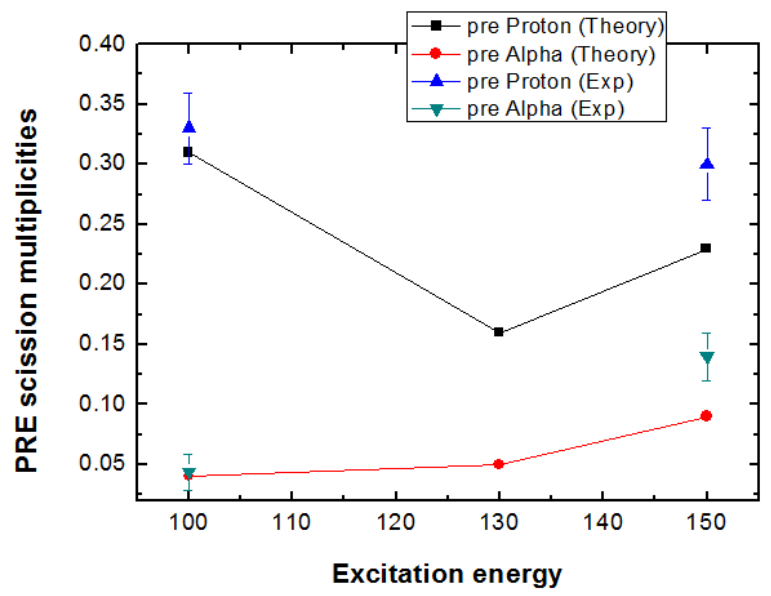

Figure 3. Pre-scission proton and $\alpha$ particles multiplicities measured for the system ${ }^{19} \mathrm{~F}+{ }^{106} \mathrm{Cd}$ compared to the full one-body dissipation dynamical calculations assuming no temperature dependence.

model for different excitation energies, a dependence on the temperature must be taken into account considering the features of the deviation.

In the present contribution we report on the preliminary results of an experiment performed at Laboratori Nazionali di Legnaro using the $4 \pi$ array $8 \pi$ LP. For the system ${ }^{19} \mathrm{~F}+{ }^{106} \mathrm{Cd}$, at two lab energies 175 and $195 \mathrm{MeV}$, we have measured most of the relevant quantities in the evaporation residues (ER) and fission channel (FF) channels: proton and $\alpha$-particle energy spectra and multiplicities, ER and FF cross sections, as well as mass and total-kinetic-energy distributions of fission fragments. The extraction procedure of the proton and $\alpha$-particle multiplicities from the energy spectra measured in coincidence with ER and FF is the same as in Refs.[8, 15]. Modeling of the fission process has been performed using again the same model as above.

In figure 3 we show the pre-scission protons and $\alpha$-particle multiplicities compared to the model results. We observe that at lower excitation energy, the model reproduces the experimental data, whereas at the higher excitation energy the experimental multiplicities are underestimated. This kind of trend suggests that a stronger viscosity would be necessary at higher temperature.

\section{Conclusions}

The analysis presented here remarks how promising is the dynamical approach in describing both fission and ER channel within the same model. In this contribution we have proposed an alternative scenario where the study of the dependence of the nuclear viscosity from the temperature seems more suitable. From the preliminary results, it seems that nuclear viscosity is slightly dependent on the temperature. However, this conclusion would indeed need further checks because the comparison with the model is so far limited to only two observables. A more stringent conclusion can be drawn after analyzing all together also the other observables still to be extracted from the experimental data.

\section{References}

[1] D. Hilscher and H. Rossner, Ann. Phys. (France) 17, 471 (1992), and references therein 
[2] P. Paul and M. Thoennesen, Ann. Rev. Nucl. Part. Sci. 44, 65 (1994), and references therein

[3] B. B. Back et al. Phys. Rev. C 60, 044602 (1999)

[4] A. Chatterijee et al, Phys. Rev. C 52, 3167 (1995)

[5] I. Dioszegi et al., Phys. Rev. C 61, 024613 (2000)

[6] G. La Rana et al., Eur. Phys. J. A 16, 199 (2003)

[7] E. Vardaci et al. Eur. Phys. A 43, 127 (2010)

[8] A. Di Nitto et al., Eur. Phys. J. A 47, 83 (2011)

[9] E. Vardaci et al., EPJ Web Conf. 62, 07004 (2013)

[10] T. Wada et al., Phys. Rev. Lett. 70, 3538 (1993)

[11] P. Frobrich and I. I. Gontchar, Phys. Rep. 292, 131 (1998), and references therein

[12] A. V. Karpov et al., Phys. Rev. C 63, 54610 (2001)

[13] P. N. Nadtochy et al., Phys. Rev. C 65, 64615 (2002)

[14] P. N. Nadtochy et al., Phys. Lett. B 685, 258 (2010)

[15] E. Vardaci et al. Phys. Rev. C 92, 034610 (2015)

[16] M.G. Itkis, E. Vardaci, I.M. Itkis, G.N. Knyazheva, E.M. Kozulin Nucl. Phys. A 944, 204 (2015) and references therin

[17] J. Cabrera et. al, Phys. Rev. C 68, 34613 (2003)

[18] E. Mordhorst et al., Phys. Rev. C 43, 716 (1991) 